\title{
GNREL
}

\section{Quantifying the Mobility and Energy Benefits of Automated Mobility Districts Using Microscopic Traffic Simulation}

Lei Zhu, Venu Garikapati, Yi Hou, and Stanley Young, National Renewable Energy Laboratory

Yuche Chen, Vanderbilt University; H. M. Abdul Aziz, Oak Ridge National Laboratory

AUTOMATED MOBILITY DISTRICTS (AMDS)

- District-scale implementation of advanced vehicle $(\mathrm{AV})$ technologies Full benefits of an AV-shared-mobility service (SAE Level 5) within confined geographic region or distric

AMD model serve "special genertos" in airports, university campuse AMD modeling and simulation toolkit informed by real-world deployments to quantify the mobility and energy impacts of an AMD
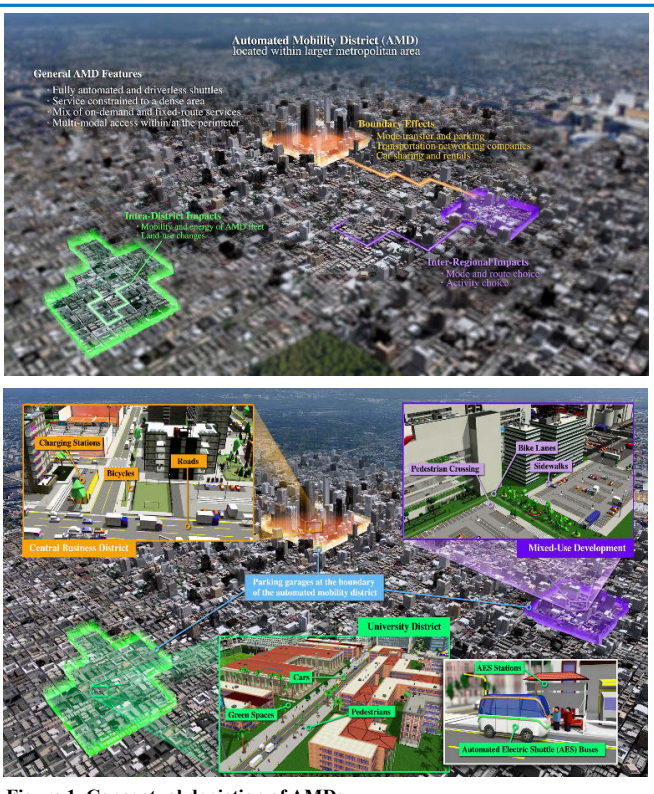

Figure 1. Conceptual depiction of AMDs

MODEL DESCRIPTION

AMD toolkit

Builds on the Simulation of Urban Mobility (SUMO)-a leading open- source microscopic traffic simulation suite

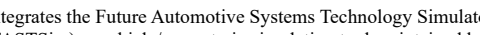

.

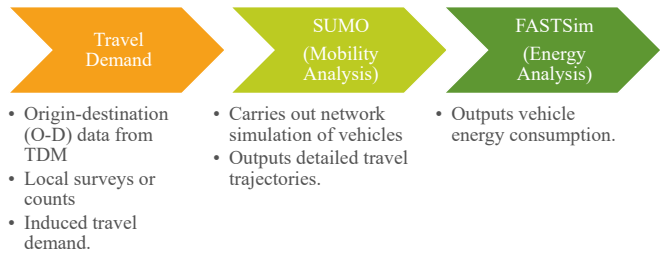

Figure 2. AMD simulation toolkit: model flow
CASE STUDIES

A Hypothetical AMD Scenario

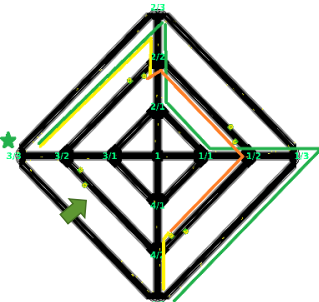

Four AES vehicles operate "on-

The simulation is carried out for demand of 300 trips distributed across the 13 (O-D) pairs. Traffic demand is distributed as a bimodal distribution reflecting a during a typical day.

The choice set of travel modes encompasses: 1) passenger car, 2

Figure 3. A hypothetical AMD network in SUMO Green lines $\rightarrow$ Car mode Red lines $\rightarrow$ AES mode

\section{Scenario}

mode

$\begin{array}{llll}\text { VMT } & \text { VATT } & \text { VATD } & \text { FC (gal) Igasoline }\end{array}$

\begin{tabular}{lllll}
\hline Baseline (70:30:0) & 128.8 & 86.5 & 0.6 & 5.9 \\
\hline
\end{tabular}

\begin{tabular}{|lllll}
\hline Transitional $(60: \mathbf{2 0}: \mathbf{2 0})$ & 153.8 & 124.3 & 0.8 & $7.0 / 5.3$ \\
\hline Optimistic $(\mathbf{5 0}: \mathbf{1 0}: 40)$ & 175.7 & 168.5 & 1.1 & $8.0 / 4.5$
\end{tabular}

Vehicle miles traveled (VMT); vehicle average travel time (VATT); vehicle average travel
distance (VATD): fuel consumption (FC) in gallons of gasoline

Mobility impact: compared to the baseline scenario, the transitional and

optimistic scenarios exhib

For the gasoline automated shuttle case, total gasoline consumption

increases along with total VMT.

For the AES case, the transitional and optimistic scenarios see fuel

consumption decrease by $10 \%$ and $26 \%$, respectively.

Kansas State University Campus (Manhattan, KS)

Kansas State University campus considered using a personal rapid transit (PRT) system to provide last mile mobility on campus
Area: 664 acres; population: $\sim 25,000$.
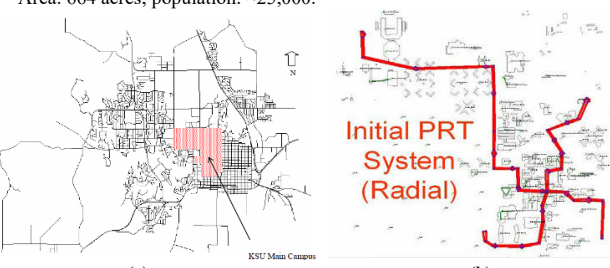

Figure 4. Kansas State University campus: (a) PRT system and (b) test region in satellite image

Analyzed mobility metrics (personal miles traveled [PMT]/personal hours traveled [PHT]) w

- 10 AMD miles displaced $\sim 4.5$ ped \& 4.5 vehicle miles

- 4 AMD hours displaced $\sim 3$ ped \& 1 vehicle hour

- Total system VMT increased $2 \%-3 \%$

Toule will travel further to go faster)
Greenville AMDs
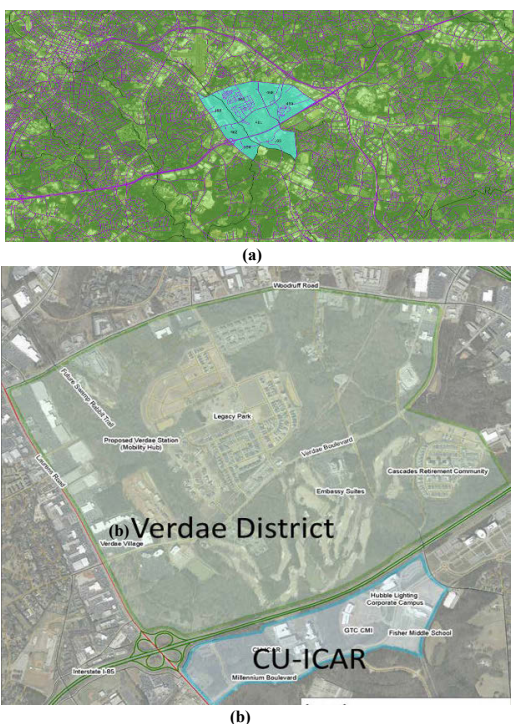

Figure 5. Greenville AMD automated taxi (a-taxi) shuttle service test region: (a) detailed road network and traftic

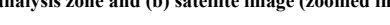

On-demand fixed route automated shuttles (a-taxi shuttles) are operating inside Greenville field test region (AMD)

traffic demand and network data (Figure 4(a) from Greal-world

County

AMD is developed for mobility and energy assessment

Multi-modal traffic flow: 1) passenger car, 2) walking, 3) on-

demand fixed-route shuttles, 4) on-demand door-to-door rid-

The Grectivis

\section{RESULTS AND DISCUSSION}

An AMD is a district-scale implementation of connected and automated vehicle technologies to realize the full benefits of an odemand shared automated mo

cegypothetical AMD a

A introduced.

This study develops an AMD modeling and simulation toolkit and reports on the preliminary analysis results for hypothetical AMD deployment, exercising the tookit with three scenarios. Future research will focus on enhancing the toolkit to integrate various performance metrics for AMDs. The modet will alo be extended tocont

issues (avalbitity and access times). 